\title{
STUDIES OF THE RETINAL CAPILLARIES IN RELATION TO DIABETIC AND OTHER RETINOPATHIES*
}

\author{
BY \\ NORMAN ASHTON \\ Department of Pathology, Institute of Ophthalmology, University of London
}

ONLY 10 years ago laboratory research on the problem of diabetic retinopathy was confined to a few investigators working in isolated ophthalmological centres, but to-day it is attracting widespread attention from many different quarters and the literature is alive with new observations and new theories. It is therefore timely to consider to what extent the various pathological features of the disease may be specific for diabetes or simply fundamental reactions to non-specific injury. I should like to consider some recent findings from my own and other laboratories which throw further light on normal and abnormal reactions of retinal capillaries, and to discuss their possible relation to the pathological picture of diabetic retinopathy and to some of the theories of pathogenesis which have been advanced.

Three of its most important manifestations will be discussed, namely endothelial proliferation, capillary microaneurysms, and capillary closure. The question of venous dilatation is a clinical one, and there is still not complete agreement that it is an early sign of diabetic retinopathy, and I shall not therefore deal with it as a pathological entity. Everyone would surely agree that the exudates are of only secondary importance. Before discussing these three pathological manifestations, a few comments will be made on our technical methods and upon the normal retinal capillaries.

\section{Methods}

Injection techniques and special staining methods are of great value in studying the normal and morbid anatomy of the retinal vessels, especially when the findings are considered in relation to preparations in which the vessels have been isolated from the retina. Injection particularly provides valuable evidence of the extent and distribution of capillary closure - a pathological aspect of diabetic retinopathy which has been much neglected. In studies of the ocular circulation in my laboratory, vessels have been isolated in the past from the retina by agitation of the unfixed tissue-these we called "shake" preparations (Ashton, 1949). Neoprene casts of retinal vessels were separated by corrosion with acids and alkalis, and casts of Schlemm's canal, of the retina, and of choroidal vessels were freed of tissue by an original technique wherein the tissues were digested with pepsin and trypsin (Ashton, 1951a; 1952; 1953; Ashton and Smith, 1953; Wybar, 1954a, b). It has only recently been recognized, however, that the retinal vessels themselves are

* Paper given at a joint meeting of the Irish Ophthalmological Society and the British Diabetic Association held in Dublin, April 25 to 27, 1963. 
relatively resistant (or the retinal tissue relatively sensitive) to the action of trypsin, so that at a critical point in digestion the retinal vessels may be isolated intact. For this discovery, which is providing major advances, not only in our understanding of the pathology of vascular retinopathies but also of endothelial reactions as a whole, we are indebted to Dr. T. Kuwabara* who, with his colleagues, has reported, in a series of papers, a number of remarkably interesting findings (Kuwabara and Cogan, 1960; Toussaint, Kuwabara, and Cogan, 1961; Kuwabara, Carroll, and Cogan, 1961; Cogan, Toussaint, and Kuwabara, 1961; Toussaint, Cogan, and Kuwabara, 1962; Reinecke, Kuwabara, Cogan, and Weis, 1962).

In the following studies, however, we have used our original method of pepsin and trypsin digestion, which dispenses with the need to wash the specimen for 24 hours. It differs from Kuwabara's technique in that the formalin-fixed retina after rinsing in distilled water is transferred immediately to 3 per cent. pepsin in 1 per cent. hydrochloric acid, incubated at $37^{\circ} \mathrm{C}$ for $20-30$ minutes, and then washed in distilled water before being transferred to the 3 per cent. trypsin solution.

\section{Normal Retinal Capillaries}

In digest preparations of the normal human retina stained with haematoxylin and eosin, the capillaries appear as perfectly regular eosinophilic tubes 7 to 8 microns in diameter. Situated at fairly regular intervals of about 50 microns are the large oval pale-staining nuclei of the endothelial cells lying parallel to the long axis of the vessels. Equally regularly disposed are the pericytes, with rounded hyperchromatic nuclei, apparently lying, usually transversely, on the surface of the capillary. Electron microscopy, however, shows these cells to be embedded in basement membrane (Kisch, 1957; Maeda, 1958; Kissen and Bloodworth, 1961; Missotten, 1961; Ishikawa, 1963), and they have been termed "mural cells" by Kuwabara and others (1961) - a term not yet adopted here as it implies a type of cell peculiar to the retina, whereas they are very similar to those found in the nervous system elsewhere and may be identical (Farquhar and Hartmann, 1957; Maynard, Schultz, and Pease, 1957; Donahue and Pappas, 1961). Cogan (1963) believes that pericytes of the type seen in the retina are unique in this tissue, justifying a special name: there may be quantitative or qualitative differences and further investigation of this important point is required.

Electron microscopy shows the pericyte to resemble a smooth muscle cell (Hogan and Feeney, 1963; Ishikawa, 1963); in fact, in digest preparations, the muscle cells of the precapillary arteriole can be seen to merge imperceptibly into the pericyte of the capillary. Moreover, it has been shown that the pericyte has a different enzyme activity as compared with that of the endothelial cell and this pattern of activity is suggestive of a smooth muscle derivation (Landers, Chason, Gonzalez, and Palutke, 1962). This would seem to indicate that the pericyte, if not actually contractile as postulated by Cogan (1962), may be responsible, at least in part, for capillary tone. The

\footnotetext{
* Dr. T. Kuwabara kindly demonstrated his technique to us some months before its publication, thus providing us with a privileged opportunity of benefiting from his work.
} 
endothelial cell, however, also exhibits tone (Zweifach, 1961a), and it is not yet clear what other specific functions these two capillary cells may have, but there would seem little doubt that variations in tone must play an important role in retinal capillary disease.

Digest preparations stained by Wilder's silver stain show a dense reticulate pattern, presumably consisting of glial processes and representing the "limitans perivascularis" described many years ago by Krückmann (1917) and more recently by Wolter (1957), which has been demonstrated with the electron microscope (Hogan and Feeney, 1963; Ishikawa, 1963). It surrounds all vessels and becomes somewhat coarser around the capillaries.

Many other features of the normal retina as seen in digest preparations have already been well described by Kuwabara and Cogan (1960); Toussaint and others (1961), and Toussaint (1961). With regard to the nature of the intercapillary bridges which they discovered stretching between the nucleated regions of capillary walls and most frequently in the retinal periphery, our own studies indicate that these are obliterated or obliterating capillaries which have become defunct through the process of retraction which accompanies normal vascular development (Ashton, 1961) or through redundancy at a later stage, or through some pathological process (e.g. long-standing injury as shown by Kuwabara and others, 1961). Capillary retraction in the developing retina leaves only a delicate strand remaining, since little or no basement membrane is present at this stage, whereas in the mature retina the collapsed basement membrane remains as a definite PAS-positive bridge. These are probably identical with the "mesodermal bridges" described by Wolter $(1957$; 1961) who stressed their possible significance in the genesis of micro-aneurysms (Wolter, 1962).

Endothelial Growth and Oxygen Tension.-There is considerable evidence to show that the growth of retinal vessels, and therefore, of course, of the endothelial cells, is very closely related, directly or indirectly, to oxygen tension in the tissues, and I have enumerated this evidence in detail elsewhere (Ashton, 1957). It is strikingly expressed in the presence of a capillary-free zone around the arteries which is present in both the developing and the adult retina of man and animals, and which can be modified in the immature animals by altering the ambient oxygen concentration. The comment by Toussaint and others (1961) that they are not "impressed by the allegedly greater concentration of capillaries about veins said to serve a supposed oxygen deficit" hardly does justice to the large amount of evidence provided by others that this is a true relationship. In injected preparations cleared with glycerol in which the normal anatomical relationships are maintained, the periarterial capillary-free zone around the artery-considerably more pronounced than around the vein-can be very readily demonstrated.

Digest preparations have provided further evidence that oxygen concentration is related to endothelial proliferation. In the developing retina 
of the kitten, for instance, it can be shown that the immature capillary network is quite unlike that of the adult cat (this is also true of the human foetus in relation to adult man). The vascular network comprises a pattern of polygonal spaces, usually pentagonal, which are largest on the arterial side of the capillary circulation and gradually diminish towards the venous side, so that in the immediate vicinity of the vein the meshwork is barely discernible and the mass of endothelial cells fuses imperceptibly with those on the venous wall. Moreover, the number of endothelial cells is notably fewer in capillaries adjacent to an artery, where they are in a single layer around the lumen, as compared with capillaries on the venous side, where they may be more than double this number (Figs 1,2,3). These appearances could be interpreted as evidence of a very delicate relationship between endothelial growth and oxygen tension (or factors related to oxygen tension).

It will probably be many years before the exact mechanism of this relationship is uncovered, but there is undoubtedly here a fundamental process governing endothelial growth which can be demonstrated not only in the developing retina but also in the experimental animal and in naturally occurring disease.

\section{Endothelial Proliferation}

Endothelial Migration.-In digest preparations an increase in endothelial nuclei has been accepted as evidence of endothelial proliferation, but there is a most misleading phenomenon to be taken into consideration, namely, that endothelial cells can readily become detached from their basement membrane and slide along the capillary lumen, so that they aggregate in clusters and closely resemble proliferations from which they are sometimes indistinguishable. The pericytes, on the other hand, being fixed in the basement membrane, are unable to move in this way; and this provides a clue in differentiating migration from proliferation, for if, in an area of apparent proliferation, the adjacent capillaries are devoid of endothelial cells yet retain their pericytes, it is possible that the appearances are due to endothelial migration and not to proliferation (Fig. 4).

It is important to make this distinction if possible for it would appear that migration has already been misinterpreted as endothelial loss or endothelial proliferation. With regard to the absence of endothelial cells, Kuwabara and others (1961) have described a selective loss of endothelial cells in capillaries with normal pericytes, and have concluded from this appearance that the pericyte survives longer than the endothelial cell, whereas it is more probable that the endothelial cells have simply migrated from these vessels. Conversely, clustered migrated cells have been erroneously described as proliferation by Reinecke and others (1962) in experiments on retinal ischaemia in cats. (Their Figs $C$ and $D$ show typical endothelial migration which is described as proliferation.) 


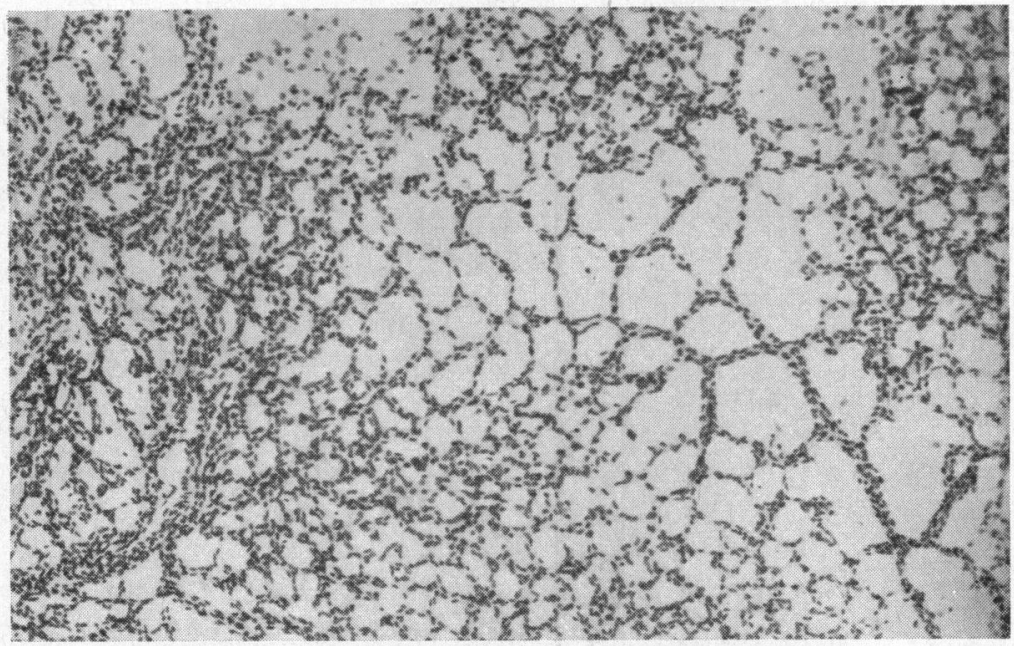

FIG. 1.-Normal developing retinal vessels of a 13-day-old kitten. Note that the capillary network is unlike that of the adult retina and consists of pentagonal spaces, which are larger towards the arterial side (right) than towards the venous side (left). Endothelial cells are fewer in the capillaries on the arterial side (cf. Figs 2 and 3). Digest preparation. P.A.S.-H (periodic acid-Schiff and haematoxylin). $\quad \times 70$.

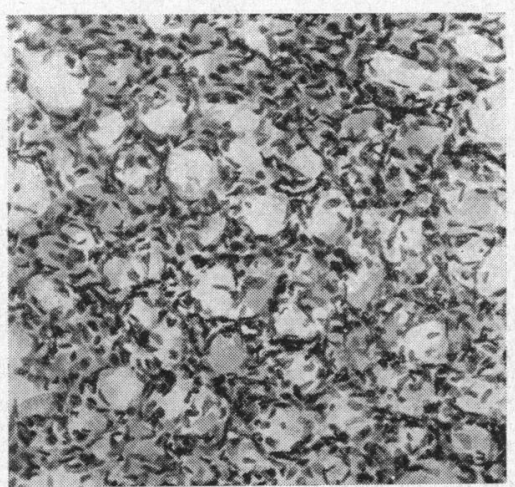

FIG. 2.-High power view of capillaries on venous side. Note small meshwork and numerous endothelial cells. Digest preparation. P.A.S.-H. $\times 280$.

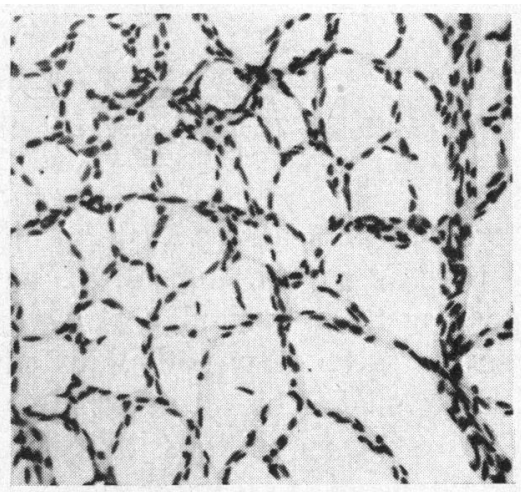

FIG. 3.-High-power view of capillaries on arterial side. Note large meshwork and small number of endothelial cells. Digest preparation. P.A.S. - H. $\times 280$.

A process similar to migration has been described in extra-ocular capillaries by Zweifach (1961a) as "endothelial desquamation". He found that repeated mechanical stimuli of endothelial cells caused them to round up and detach from adjacent cells and eventually to fall into the lumen and be swept away by the blood stream. The process I am describing differs in that the cells rather appear to slide along the lumen after detachment. Whether this is related to the active migration of endothelial cells which occurs in the normal process of "retraction" and which can be exaggerated by increasing ambient 


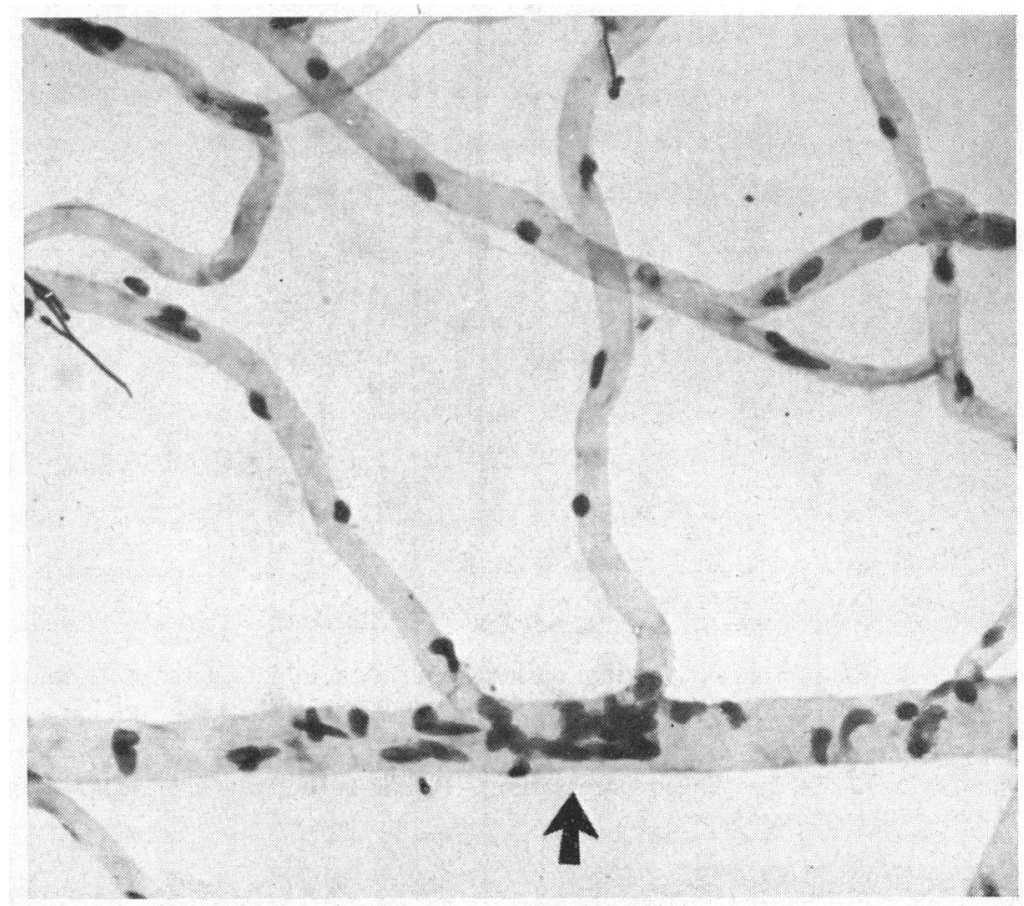

FIG. 4.-Aggregation of endothelial cells in lowest vessel (arrow), is due to migration of endothelial cells from adjacent vessels and not to endothelial proliferation. The pericytes, being intramural, remain in situ. Digest preparation. P.A.S.-H. $\times 280$.

oxygen concentrations (Ashton and Pedler, 1962) is unknown, and to what extent it is a pathological, agonal, or post mortem change has yet to be determined. It is similar, however, to the process occurring in vivo, and the cohesive state of endothelium can be altered by perfusion with calcium deficient media, by an acid pH, or proteolytic enzymes (Zweifach, 1961b); the last suggests the possibility that this may even be an artefact of trypsin digestion, and it is significant that we have been unable to find endothelial migration in retinal capillaries isolated by our earlier shake method.

Endothelial Proliferation in Disease.-In previous papers I have discussed the factors concerned in retinal neovascularization in disease (Ashton, 1957, 1961) and have emphasized the non-specific quality of this reaction, which, irrespective of the condition, appears always to be a response to the stimulus of hypoxia in the same manner as normal vascularization. The digest method now permits further examination of this question in revealing the behaviour of the endothelial cells themselves. They proliferate in two, not necessarily distinct, circumstances:

(a) Where the stimulus is intravascular. Endothelial cells will increase in number, for instance, in vessels where the circulation is sluggish (as in the 


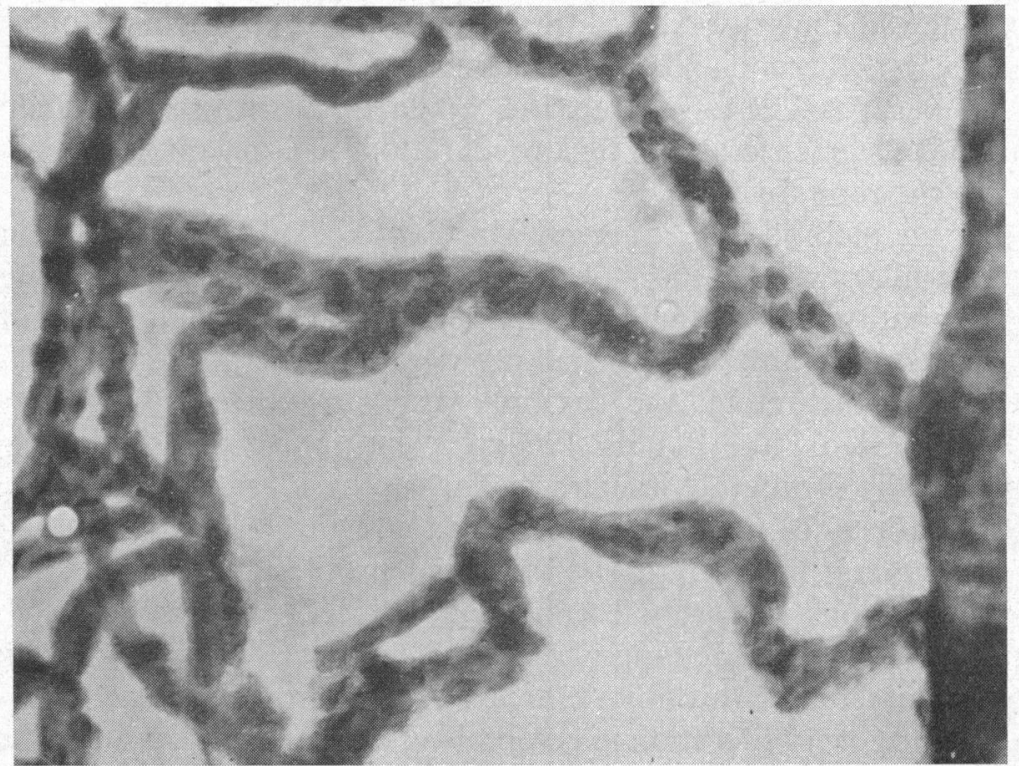

FIG. 5.-Endothelial proliferation in the retinal capillaries in a case of macroglobulinaemia. Digest preparation. P.A.S.-H. $\times 390$.

capillaries of the disc in papilloedema, and in cases of macroglobulinaemia: Ashton, 1962) (Fig. 5) or in some cases of prolonged cyanosis (as in emphysema):

(b) Where the stimulus is extravascular. Here proliferation occurs in vessels related to ischaemic retinal tissue and not infrequently this proceeds to neovascularization. It is seen, for instance, after the closure of capillaries by oxygen in the immature retina, or through thrombosis or inflammation (Eales's disease) in the mature retina. In all these circumstances hypoxia would seem to be the common factor. Complete anoxia, however, leads to the death of endothelial cells; thus no proliferation will be found in retinal arterial occlusion for here the vessels are acellular, and the same is true in capillaries which have been closed for any length of time.

In diabetic retinopathy endothelial proliferation and neovascularization are well known features, and seem to be equally non-specific responses. They occur in engorged and dilated capillaries, and always in vessels related to areas of capillary closure - to which I shall presently refer more fully - and it is these latter areas which provide the stimulus for the formation of rete mirabile, and which sometimes contain solid masses of endothelial cells.

Endothelial Proliferation in Cerebral Vessels.-The remarkable degree of endothelial proliferation in the retina in diabetes naturally prompts the enquiry whether similar changes occur in the body elsewhere. It is unfortunate that the digest method for isolating vessels works satisfactorily only in the retina; in other tissues we have examined (choroid, brain, kidney), the 
vessels digest at the same rate as the stroma, and this does not appear to be due to the presence of collagen as stated by Toussaint and others (1961). It is, however, possible to separate the capillaries of the brain by shaking the fresh tissue in saline, so obtaining a preparation, less elegant, but comparable to that of the retinal vessels.

Using this method, my colleague Dr. Taktikos and I have examined the cerebral capillaries in 26 cases of diabetes with severe retinopathy and have found no abnormality. Similarly, in a case of macroglobulinaemia wherein there was considerable endothelial proliferation and thousands of microaneurysms in the retina, the cerebral vessels appeared normal (Ashton, 1962; Ashton, Kok, and Foulds, 1963). We know, of course, that endothelial cells readily proliferate in extra-ocular capillaries in a variety of circumstances; for instance, in cerebral anoxia (a comparable situation to the one we are discussing in the retina) endothelial proliferation has been demonstrated in sections in cerebral infarction induced experimentally (DennyBrown, Horenstein, and Fang, 1956) and we have found it in capillaries adjacent to a cerebral thrombotic area in shake preparations.

The absence of proliferation in cerebral vessels in subjects with retinopathy provides further evidence of the significance of local factors within the eye and is in line with our previous findings that microaneurysms are likewise confined to the retina.

\section{Capillary Microaneurysms}

In the past we have devoted much time in my laboratory to the study of retinal microaneurysms and at different stages of this work have been impressed by various factors in relation to them, such as venous engorgement, arteriolar occlusion, circulatory anoxia, mucopolysaccharide and lipid deposits, and tissue swelling, but with the acquisition of new knowledge and the development of new techniques, as in the application of electron microscopy and the digest method, it has become necessary to review some of our earlier conclusions.

I think it remains true that, as we originally showed, the capillary microaneurysm is one of the commonest lesions of the retinal vessels, occurring in a host of unrelated conditions. They are usually most numerous in diabetes but this is not necessarily a specific point, but may be merely an indication of the extent of the underlying disease; in fact, they are sometimes seen in non-diabetic conditions in as great or even greater profusion. Venous thrombosis is an exaniple, and in the case of macroglobulinaemia previously referred to, they were more numerous than we have seen in any other condition, the findings in this particular case illustrate well the non-specificity of the lesion (Ashton and others, 1963).

In previous work we have attached some significance to the presence of fat and mucopolysaccharides in the aneurysmal wall, especially in relation to nodular glomerulosclerosis, where similar changes are found, and even 
recently fat emboli have been held responsible for the development of diabetic microaneurysms (Pope, 1960). It is, therefore, interesting to find that the diabetic aneurysm in fact possesses no entirely specific features, for non-diabetic aneurysms, as seen for instance in hypertension, venous thrombosis, or macroglobulinaemia, may show an exactly similar lipoid and hyaline infiltration, although usually of a lesser degree. This infiltration, therefore, would appear to be rather a secondary development consequent upon increased permeability at these points. Similarly the binding of fluorescent insulin in capillary aneurysms, which has recently been demonstrated by Coleman, Becker, Canaan, and Rosenbaum (1962) might also be an expression of this abnormal permeability, in this case to globulin, rather than evidence of an immunological mechanism in the pathogenesis of the lesion.

Similarly, the endothelial proliferation seen so frequently within aneurysms is more likely to be a secondary manifestation and may arise in three ways: from circulatory stagnation within the aneurysm, or from increased traffic of nutritional substances through the wall, or, more especially, from endothelial migration into the aneurysm, for frequently the adjacent capillaries are denuded of endothelial cells (Figs 6 and 7, overleaf). Moreover, it is also common to find white blood cells trapped in aneurysms (Fig. 8, overleaf) and no one would claim that these cells had any significance in their pathogenesis. In our case of macroglobulinaemia, microaneurysms were most numerous at the periphery where there were no surviving cells in the capillaries, and, conversely, at the equator, where endothelial proliferation was profuse, microaneurysms were few. Thus there is no constant relationship between endothelial proliferation and microaneurysms, although they are frequently associated. I think, therefore, that we now have enough evidence to set aside the idea that microaneurysms are arrested new-vessel formations (Ashton, 1951b, Wise, 1957) or that endothelial proliferations can in any other way be incriminated in their formation (as suggested by Hausler and Sibay, 1961).

Most of us would agree that the microaneurysm is a diverticulum in the capillary basement membrane, as has been well shown recently by Cogan and others (1961) and by Bloodworth (1962), but both these groups of workers have doubted the significance of the varicose loop formations I described in diabetes. This is partly because these minute loops are clearly seen only in injected preparations, which these authors have apparently not studied, and in which numerous varicose loops may regularly be seen in diabetes (Fig. 9, overleaf), and partly because I had suggested that the majority of diabetic microaneurysms arose in this way, whereas it is now apparent that this is true only in some cases; a diverticulum may arise on one side of the capillary without a preceding loop formation. A selective destruction of pericytes has been thought to be of fundamental importance in diabetes (Cogan and others, 1961), and it is true that these cells are markedly degenerate in diabetic retinopathy. In our experience, however, this process is variable, and 


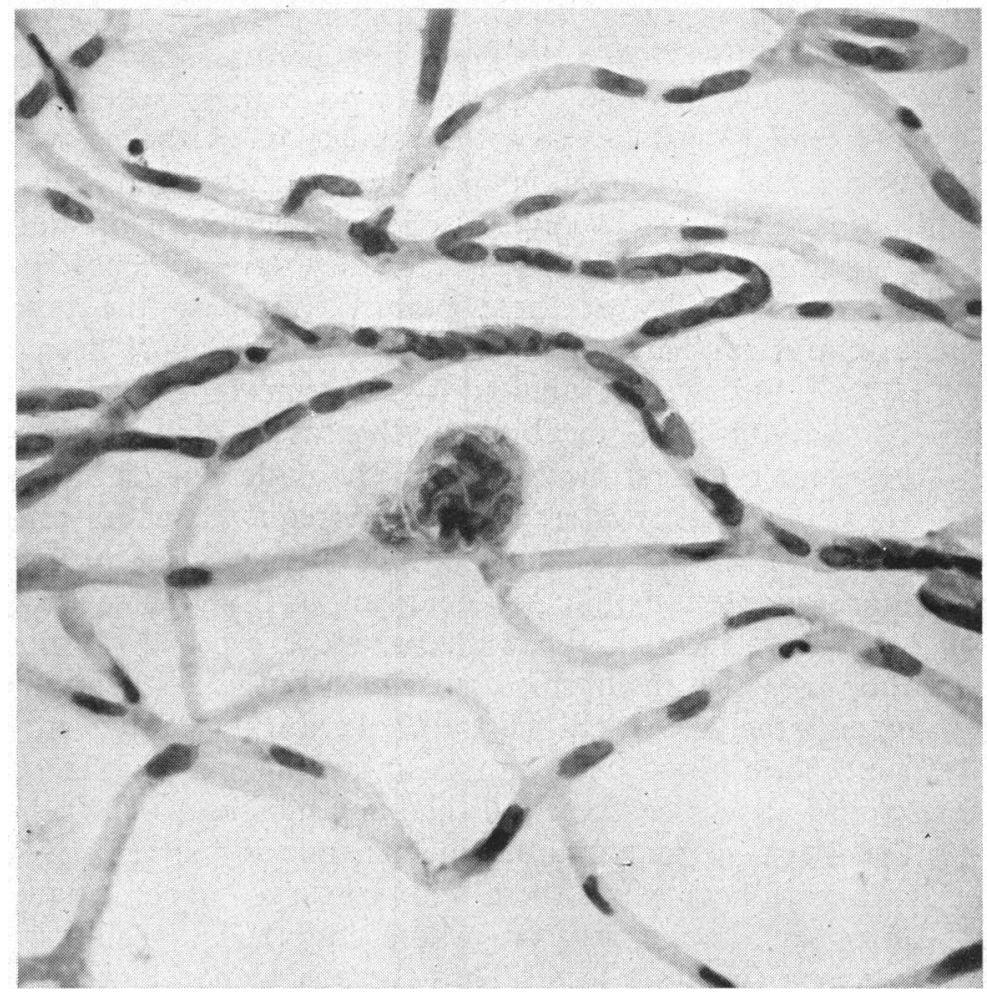

FIG. 6.-Diabetic retina, showing aggregation of endothelial cells within a microaneurysm. Digest preparation. P.A.S. - H. $\times 280$.

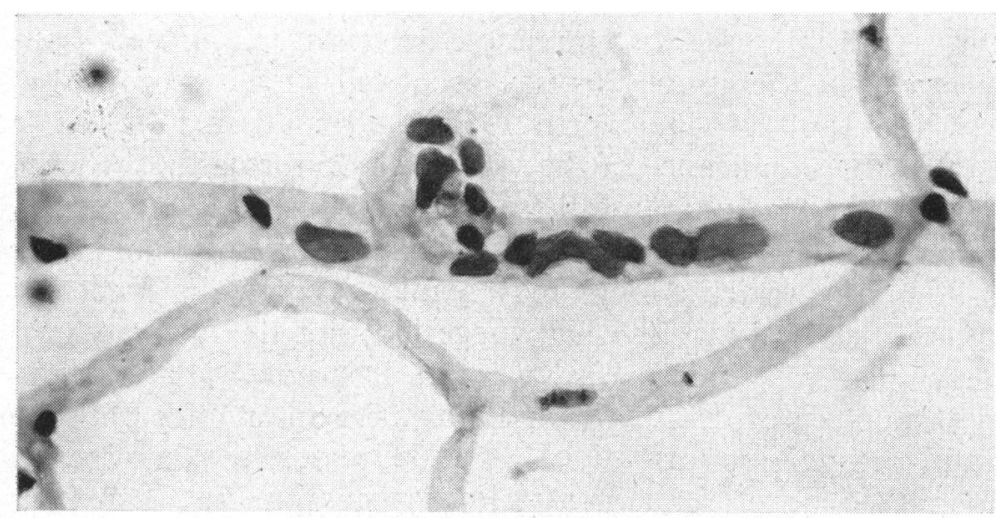

FIG. 7.-Endothelial migration in region of diabetic microaneurysm, showing how endothelial cells may come to collect within the aneurysm. Digest preparation. P.A.S.-H. $\times \mathbf{4 4 0}$. 


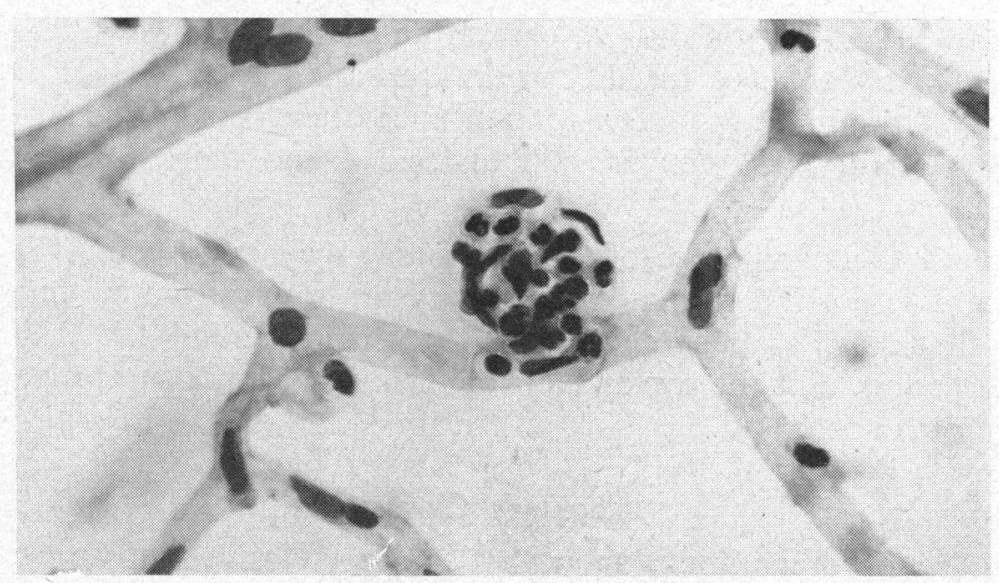

FIG. 8.-Microaneurysm in a case of venous thrombosis, showing numerous polymorphonuclear neutrophils (and endothelial cells) entrapped within it. Digest preparation. P.A.S. - H. $\times 440$.

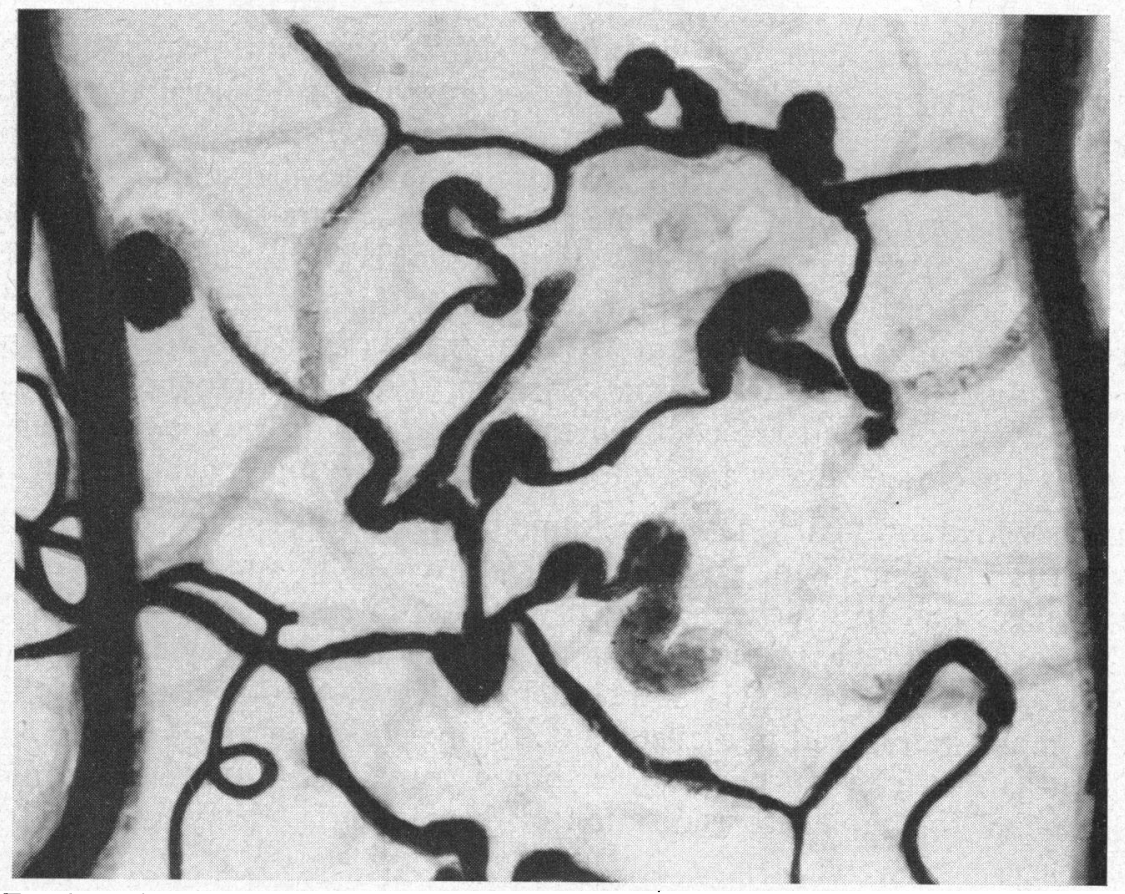

FIG. 9.-Diabetic retina, showing aneurysmal lesions on the capillaries. Note that at least eight out of twelve of these are derived from varicose loops. Injected and digest preparation. P.A.S.-H. $\times 280$. 
quite often the endothelial cells are entirely absent when pericytes still survive, nor does it appear to be specific for diabetes and, in our view, it bears no constant relationship to the formation of microaneurysms. The importance of pericyte injury is, however, still undecided and more information is required.

Whatever the significance of these minutiae may be, one must seriously entertain the possibility that microaneurysms result from nothing more specific than stasis and engorgement of the capillaries, and it is in the cause of these factors that the explanation of their appearance in any particular condition may be found.

\section{Capillary Closure}

Our injection preparations show that capillary closure is a very common pathological change in the retina, and there are good grounds for believing that this may be due to extravascular factors. To take one example, the "cotton wool" spot in hypertension is a focal area of retinal swelling usually associated with a lipo-hyaline occlusion of the neighbouring arteriole. Injected specimens show these areas to be avascular (Ashton, 1959, 1963) but, if the preparation is then digested, it will be found that the capillaries are present and apparently patent, although, in accordance with the rule that when the circulation entirely fails the cellular elements disappear, it will be found that the capillaries consist only of simple basement membrane tubes. We may, therefore, conclude that their closure is not maintained by an active constriction of the endothelial cells or of the pericytes, and it would appear that they have been compressed by the swelling of the surrounding tissue and that this has probably been facilitated by the fall in capillary pressure resulting from the arteriolar occlusion. In other words the capillary closure is a manifestation of disturbance in the normal relationship between tissue and capillary pressures. We now know that as the swelling subsides the circulation gradually returns to normal (Ashton, 1963). Microaneurysms are frequently present and are confined to the margins of these swollen areas, whereas the closed vessels are protected from aneurysmal changes.

In diabetic retinopathy, it will be found in injected specimens that capillary closure is a predominant pathological feature, which is usually focal in its early stages and to which microaneurysms are intimately related (Fig. 10). This closure can be traced in digest preparations by acellularity of the affected capillaries (Fig. 11) as noted by Cogan and others (1961). It is much more widespread in diabetes than in hypertension and becomes more evident as the disease progresses, leading eventually to virtual extinction of the capillary bed and disorganization of the capillaries themselves (Ashton, 1953).

Like capillary closure in hypertension, it bears a relationship to the arterial side of the circulation, and results in the formation of new channels 


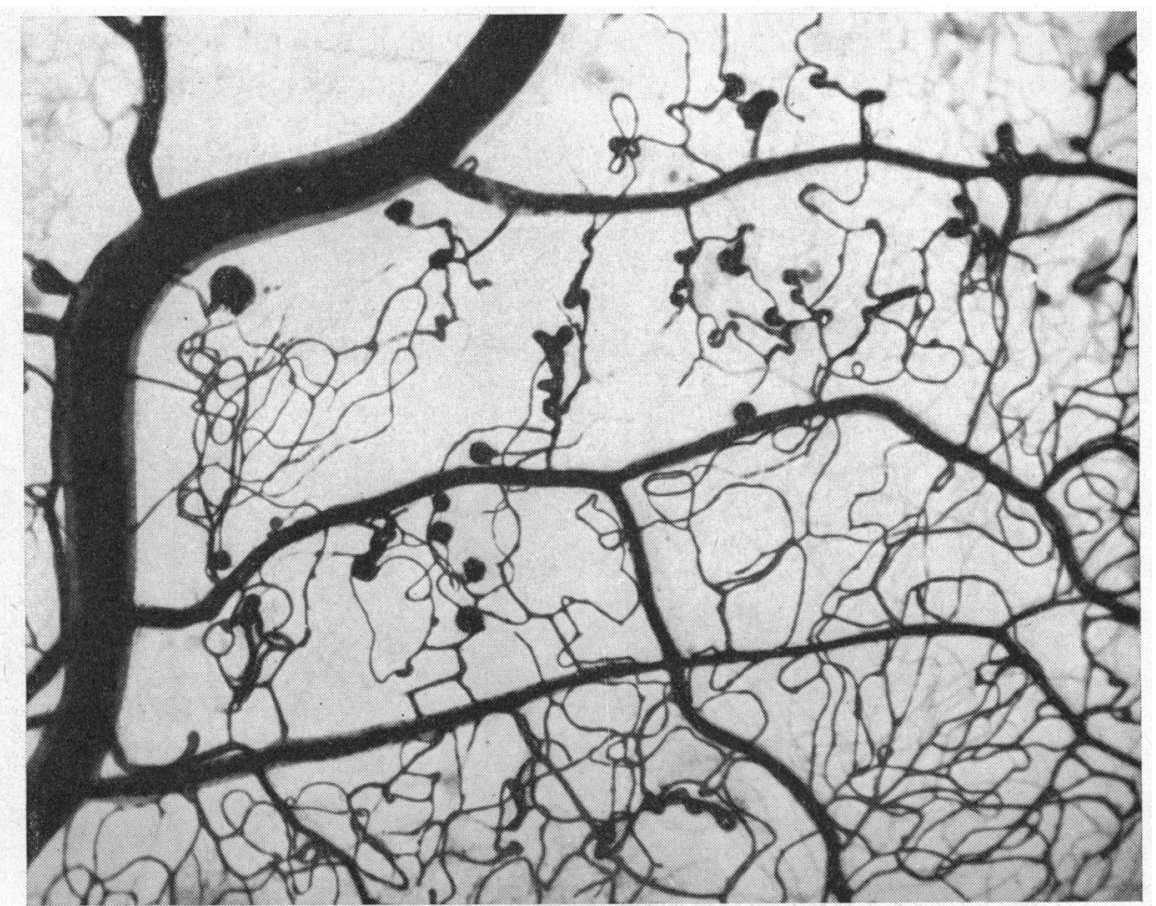

Fig. 10.-Diabetic retinopathy. Note that capillary closure (uninjected areas) is a predominant pathological feature to which microaneurysms are intimately related. Injected and digest dreparation. P.A.S. $\times 70$.

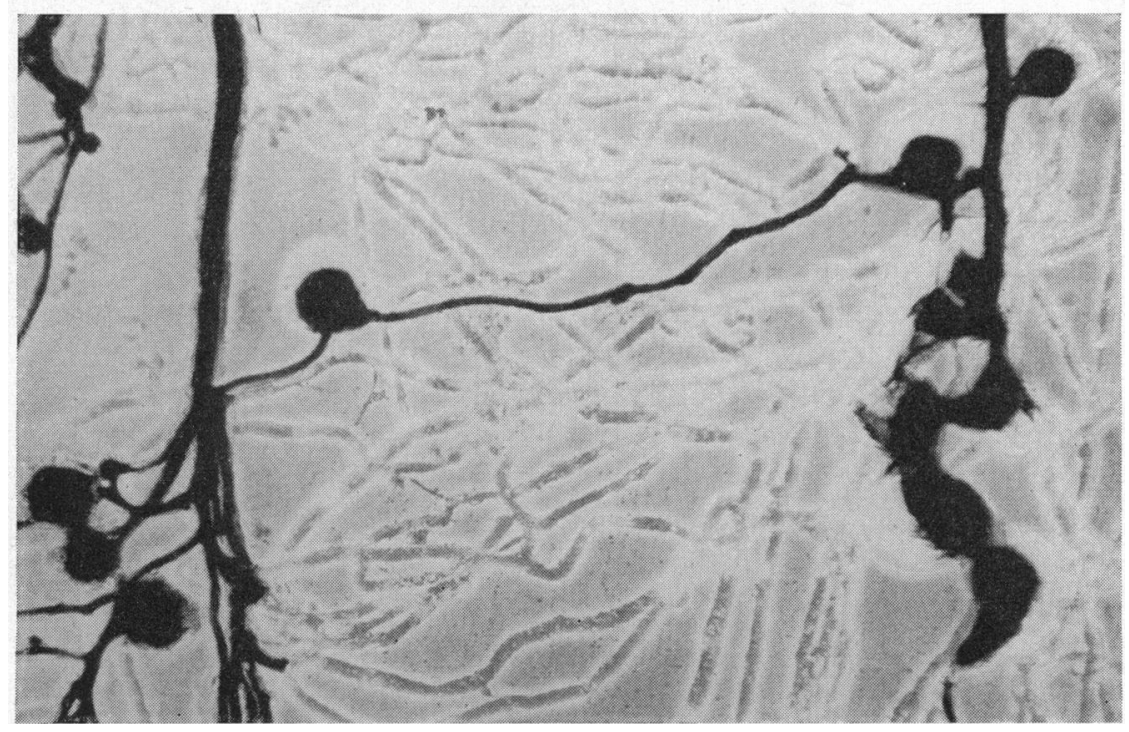

FIG. 11.-Diabetic retinopathy. The aneurysms are largely confined to patent capillaries, while the closed (uninjected) capillaries, although devoid of all cells and consisting only of basement membrane tubes, are mostly free of aneurysms. Injected and digest preparation. Phase contrast. $\times 170$. 
in the capillary network on the venous side, which become dilated and deformed to produce Y-shaped, T-shaped, and S-shaped loops bordering the avascular zones (Fig. 12).

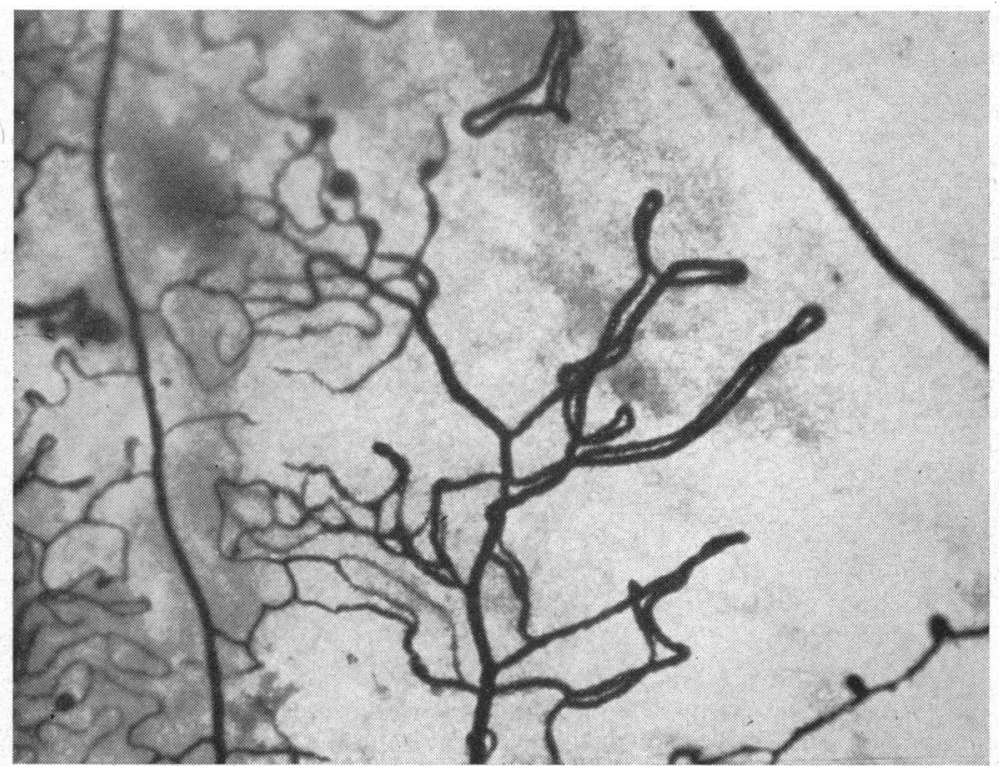

FIG. 12.-Diabetic retinopathy showing capillary closure around the artery (top right) and the formation of dilated loops in the capillary bed on the venous side (cf. Fig. 13). Injected preparation. Unstained. $\times 70$.

As I have described earlier, digest preparations reveal a reactive endothelial proliferation within the walls of these dilated channels (Fig. 13, opposite). Within and confined to these areas of capillary closure, the nerve fibres, as seen in silver-impregnated specimens, are swollen and degenerate and show numerous cytoid bodies or terminal nodes, providing a picture closely comparable to that of the "cotton wool" spot (Fig. 14, opposite).

Discovery of the cause of this capillary closure in diabetic retinopathy would, I believe, provide important clues to the pathogenesis of the disease and, since these changes are peculiar to the retina, we must search for some local mechanism inherent in the metabolism or structure of the retina, or for some general change to which the retina or vessels peculiarly react.

I had recently suggested the possibility should be considered that, as in hypertension, the capillaries might be compressed by retinal swelling due to a primary metabolic injury, and I described in some detail how this might operate (Ashton, 1959). Since that time, however, the premise upon which this concept was largely based, namely that oxygen vaso-obliteration in the 


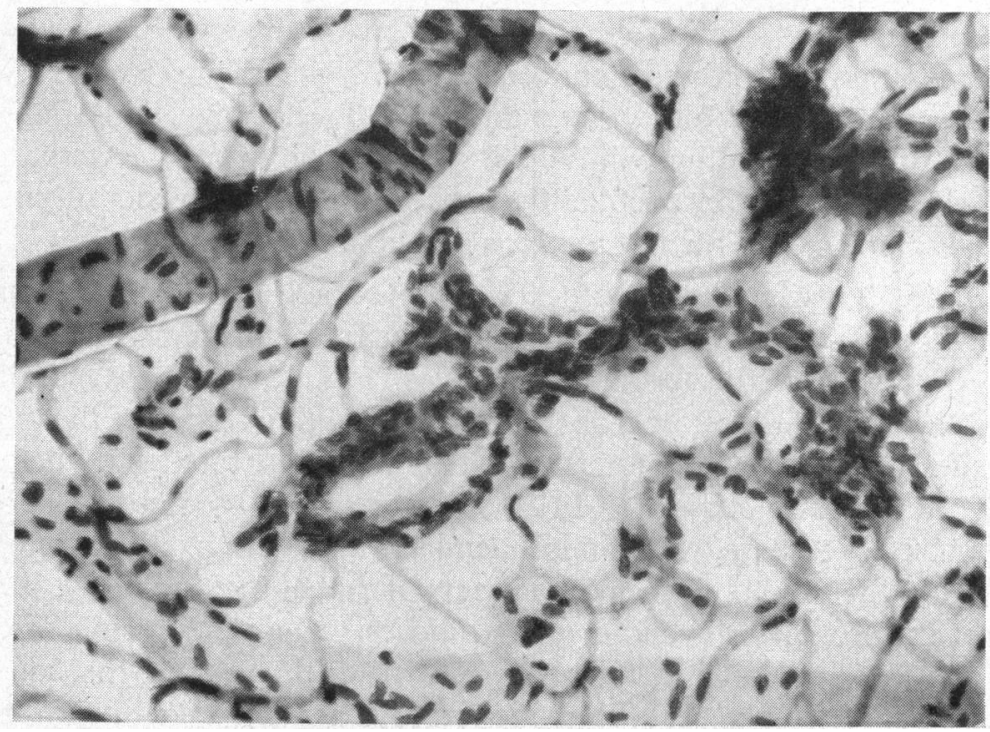

FIG. 13.-Endothelial proliferation seen within the type of dilated loop illustrated in Fig. 12. The small acellular vessels in the background are closed capillaries. Digest preparation. P.A.S.-H. $\times 170$.

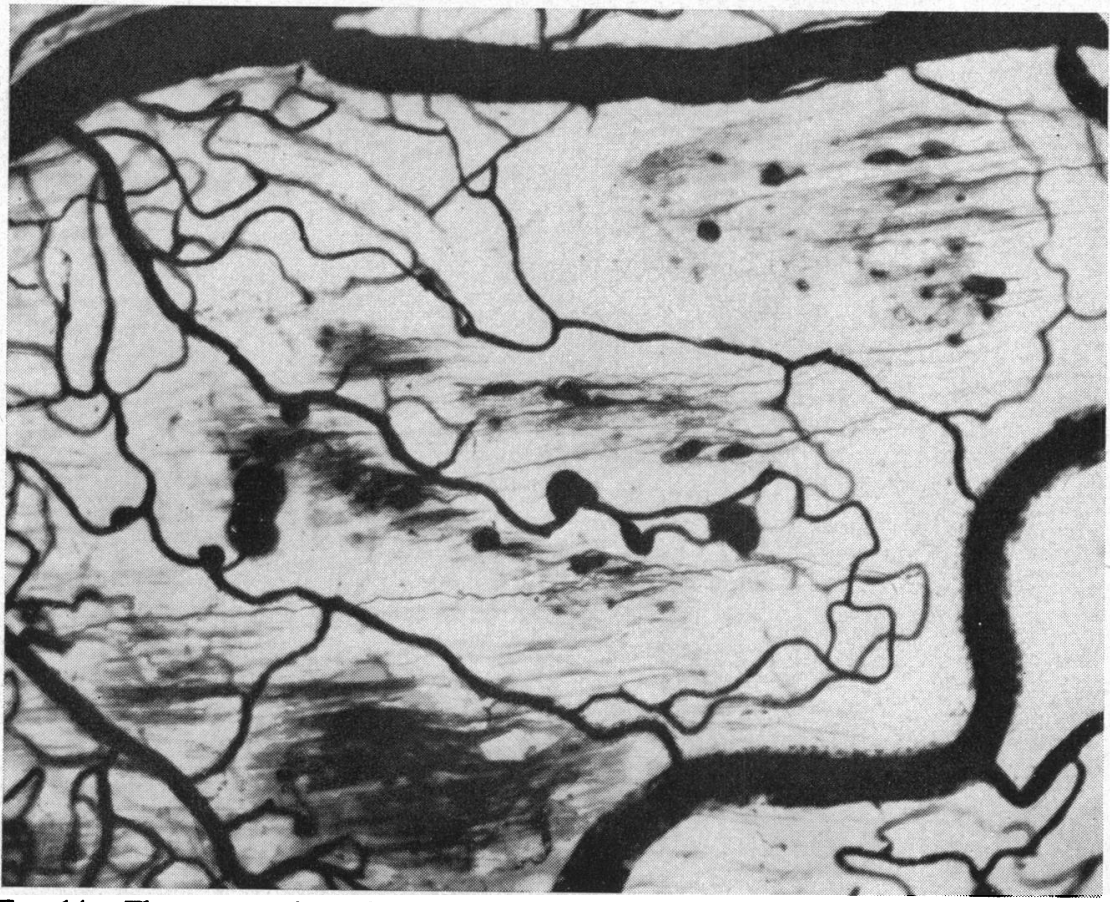

FIG. 14.-Flat preparation of diabetic retina, injected with Indian ink and stained by silver impregnation (Gross-Schultz). Degenerative changes are present in the nerve fibres which are swollen and show cytoid bodies. These changes are almost entirely confined to the areas of capillary closure, i.e. the uninjected areas. $\quad \times 110$. 
immature retina was due to retinal swelling, has been called into question by our finding that the growing endothelial cells are themselves sensitive to oxygen (Ashton and Pedler, 1962) and that developing capillaries on the surface of the rabbit retina exhibit the same phenomenon. At the present time, therefore, this approach, although still under consideration, is less encouraging and needs further analysis and re-assessment.

There are, however, many other ways in which the capillaries may be obliterated, with or without retinal swelling, as from chronic ischaemia due to gradual occlusion of the terminal arterioles (Fig. 15), perhaps purely functional in the first instance, or from a very gradual thickening of the basement membranes of the arterioles (as is now being demonstrated elsewhere in the body of the diabetic subject). In this connexion it is interesting to recall that, in pulseless disease, wherein ischaemia would seem to be the only factor concerned in the retina, all the components of diabetic retinopathy developcapillary closure, arteriovenous anastomoses, microaneurysms, and neovascularization. Indeed, at the time of writing this paper, the concept of retinal ischaemia as a cause of diabetic retinopathy has much to commend it, especially in relating it to extra-ocular vascular disease in diabetes. The hypothesis that diabetic retinopathy was primarily a disease of the venous side of the circulation may have been misleading.

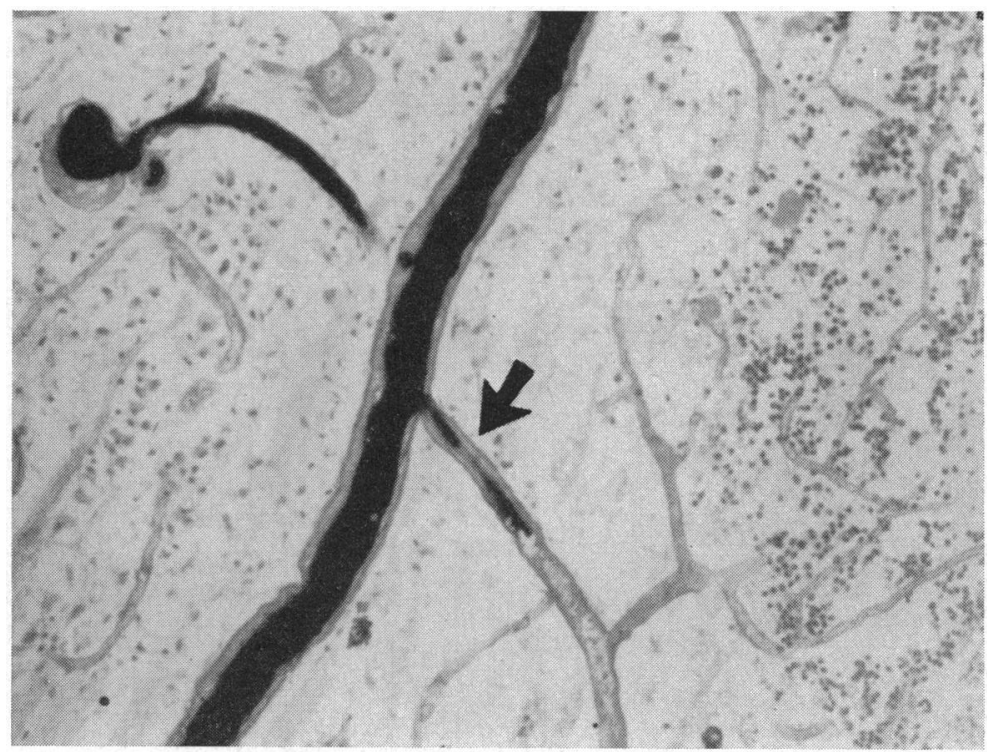

FIG. 15.- Hyaline occlusion of a terminal arteriole (arrow) in diabetic retinopathy. Many capillaries are uninjected and acellular. Flat section of injected retina. P.A.S.-H. $\times 70$. 
Among the many other factors to be considered is increased permeability of the capillaries through endothelial injury-as postulated long ago by Ballantyne (1945) -or specific pericyte injury-as suggested by Cogan and others (1961) — with loss of capillary tone and re-distribution of the capillary circulation; embarrassment of the venous outlet has also been suggested to explain both the venous engorgement seen early in the retinopathy, and the similarity between the pathology of diabetic retinopathy and venous thrombosis (Fig. 16).

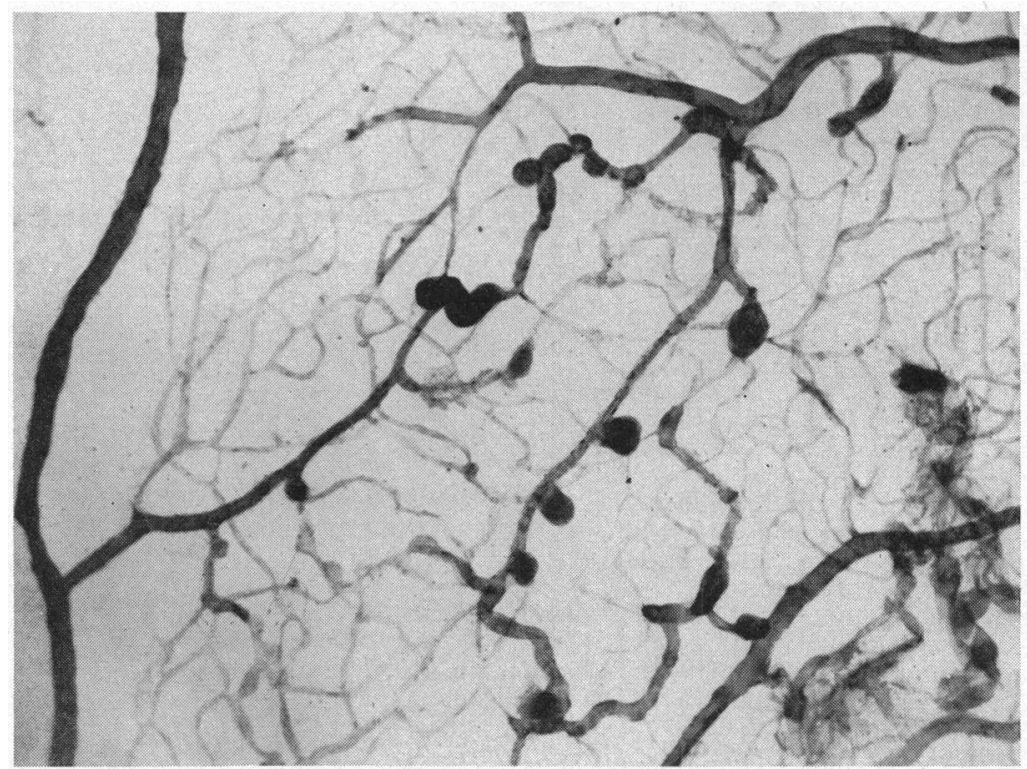

FIG. 16.-Retinal changes in venous thrombosis similar to those of diabetes. Note aneurysms on patent arteriovenous anastomoses, and the closed non-aneurysmal acellular capillaries in the background. Digest preparation. P.A.S.-H. $\times 70$.

It is clear that, although advances are being made in detailed observations of structural changes, we still have very little knowledge of their cause. Research could be more efficiently directed if it were possible to decide which features of diabetic retinopathy are specific and which non-specific for diabetes. There is little doubt that haemorrhage, exudates, and neovascularization are secondary developments, and it would seem possible that the microaneurysms may be merely manifestations of engorgement and stasis within the retinal capillaries. Investigations which attempt to explain them in terms directly related to diabetes (e.g. insulin deficiency, high glucose levels, specific immune processes, etc.) may therefore prove unrewarding.

Capillary closure, on the other hand, although itself a secondary development, appears to be a key factor, and its explanation may uncover the whole mechanism of the pathogenesis of diabetic retinopathy. It would seem, 
however, that our knowledge of the morbid anatomy of this condition is nearing completion, and future discoveries are more likely to come from dynamic studies on retinal vessels in animals and man, the value of which has been greatly enhanced by the use of intravenous fluorescein.

My thanks are due to Professor Vrabec for assistance with the silver impregnation methods, to Mr. G. E. Knight and Mrs. A. Fergusson for technical assistance, and to Miss E. FitzGerald for secretarial help.

Ashton, N. (1949). Brit. J. Ophthal., 33, 407.

(1951a). Ibid., 35, 291.

(1951b). Proc. roy. Soc. Med., 44, 747.

(1952). Brit. J. Ophthal., 36, 465.

(1953). Ibid., 37, 140.

(1957). Amer. J. Ophthal., 44, No. 4, pt 2, p. 7.

(1959). Lancet, 2, 625.

(1961). Trans. ophthal. Soc. U.K., 81, 145.

(1962). "Diabetic Retinopathy", in "Disorders of Carbohydrate Metabolism", ed. D. A. Pyke. Pitman Medical, London.

(1963). Trans. ophthal. Soc. U.K., 83 (In press).

- KoK, D'A., and Foulds, W. S. (1963). J. Path. Bact., 85 (In press).

and Pedler, C. (1962). Brit. J. Ophthal., 46, 257.

and SMITH, R. (1953). Ibid., 37, 577.

Ballantyne, A. J. (1945). Arch. Ophthal. (Chicago), 33, 97.

BloOdWORTH, J. M. B. (1962). Diabetes, 11, 1.

CoGAN, D. G. (1962). Amer. J. Ophthal., 54, 347. (1963). Ibid., 55, 159.

Coleman, S. L., Becker, B., CanaAn, S., and Rosenbaum, L. (1962). Diabetes, 11, 375.

Denny-Brown, D., Horenstein, S., and FANG, H. C. H. (1956). J. Neuropath. exp. Neurol., 15, 146.

Donahue, S., and Pappas, G. D. (1961). Amer. J. Anat., 108, 331.

Farquhar, M. G., and HartmanN, J. F. (1957). J. Neuropath. exp. Neurol., 16, 18.

Hausler, H., and Sibay, T. M. (1962). Diabetes, 10, 452.

Hogan, M. J., and FEENEY, L. (1963). Invest. Ophthal., 2, 101.

ISHIKAWA, T. (1963). Ibid., $2,1$.

KISCH, B. (1957). Exp. Med. Surg., 15, 89.

Kissen, A. T., and Bloodworth, J. M. B. (1961). Exp. Eye Res., 1, 1.

KrüCKmann, E. (1917). Z. Augenheilk., 37, 1.

Kuwabara, T., Carroll, J. M., and Cogan, D. G. (1961). Arch. Ophthal. (Chicago), 65, 708. and CogAN, D. G. (1960). Ibid., 64, 904.

Landers, J. W., Chason, J. L., Gonzalez, J. E., and Palutke, W. (1962). Lab. Invest., 11, 1253.

MAEDA, J. (1958). Acta soc. Ophthal. jap., 62, 1002.

Maynard, E. A., Schultz, R. L., and Pease, D. C. (1957). Amer. J. Anat., 100, 409.

Missotten, L. (1961). Bull. Soc. belge Ophtal., No. 129, p. 382.

POPE, C. H. (1960). Diabetes, 9, 9.

Reinecke, R. D., Kuwabara, T., Cogan, D. G., and Weis, D. R. (1962). Arch. Ophthal.(Chicago) 67, 470.

ToussaInt, D. (1961). Bull. Soc. belge Ophtal., No. 129, p. 393.

Cogan, D. G., and Kuwabara, T. (1962). Arch. Ophthal. (Chicago), 67, 42.

KuWABARA, T., and Cogan, D. G. (1961). Ibid., 65, 575.

Wise, G. N. (1957). A.M.A. Arch. Ophthal., 57, 151.

Wolter. J. R. (1957). Amer.J. Ophthal., 44, No. 4, pt 2, p. 48. (1961). Ibid., 51, 1123. (1962). Diabetes, 11, 136.

WYBAR, K. C. (1954a). Brit. J. Ophthal., 38, 513. (1954b). J. Anat. (Lond.), 88, 94 .

ZWEIFACH, B. W. (1961a). Angiology, 12, 507.

(1961b). "Functional Behaviour of the Microcirculation", p. 41. Thomas, Springfield, Ill. 